\title{
MYPE, REDES SOCIALES Y CRISIS RECESIVA MUNDIAL
}

\section{MYPE, SOCIAL NETWORKS AND RECESSIVE CRISIS IN THE WORLD}

\author{
Julio Palomino Silva* \\ Docente Asociado de la Facultad de Ciencias Contables, UNMSM
}

[Recepción: Abril de 2009 / Conformidad: Mayo de 2009]

\section{RESUMEN}

El efecto que tiene la actual crisis económicofinanciera global, en nuestro país, y el papel que debe tener la micro y pequeña empresa dentro de este contexto recesivo es fundamental, por lo tanto utilizar un enfoque de redes sociales es importante para hacer frente a la actual crisis. Las limitaciones que enfrentan las MYPE y que impiden explotar su potencial, se derivan de su limitada escala y sus débiles relaciones de articulación y colaboración que podrían ser suplidas por la creación de redes que utilicen los activos sociales preexistentes. La conclusión de tan interesante vinculación es que, en las condiciones actuales del mercado mundial, la asociación y coordinación entre las micro y pequeñas empresas es una necesidad vital.

Palabras clave: MYPE, redes sociales, crisis recesiva.

\begin{abstract}
The effect that has the current economic - financial global crisis, in our country, and the role that the micro must have and small enterprise inside this recessive context it's fundamental, So use an approach of social nets is important to face to the current crisis, the limitations that the MYPE face and that prevent from exploiting their potential, they stem from their limited scale and their weak relations of joing and collaboration that might be replaced by the creation of networks that use the social preexisting assets. The conclusion of so interesting entail is that, in the current conditions of the world market, the association and coordination between the micro and small enterprises it is a vital need.
\end{abstract}

Keywords: MYPE, social networks (Nets), recessive crisis.

* Contador Público Colegiado. E-mail, japalomino2002@yahoo.com. 


\section{INTRODUCCIÓN}

El presente artículo constituye un esfuerzo por tratar de comprender la lógica de la economía interna y una posible salida al nefasto panorama de crisis global recesiva, que ya muestra sus estragos en la economía nacional.

Así, enmarcado en este contexto desarrollé este artículo con los aportes de la Sociología, en el campo del estudio de las redes sociales, cuya dinámica de trabajo me permitió acercarme a temas nuevos y que a su vez, me arrastraron a la revisión de temas antiguos y de autores variados.

En el proceso pude reconocer las novedades y las permanencias, y revisar teorías y metodologías a la luz de los grandes cambios de nuestra sociedad y del mundo.

En lo posible he tratado de abarcar la información relativa a cada tema, lo que fue enriquecido por una exhaustiva revisión de información existente.

Así pues, espero que el presente trabajo sea un aporte al esfuerzo de renovación de ideas y propuestas que todas las ciencias —desde su ángulo particular — tratan de dar para enfrentar ese monstruo llamado "crisis económico-financiera global".

Este artículo no está llamado a ser una versión total de lo propuesto, pero sí la síntesis ordenada de una propuesta a partir de las grandes preocupaciones de nuestra economía y sociedad actuales.

Lo considero un aporte también, pues implicó tomar a otra ciencia para poder sustentar mejor su contenido, y he tratado de emplear un lenguaje extremadamente sencillo para su comprensión.

Por ello, quisiera precisar que el tema tratado, me ha resultado particularmente sugerente y espero que también a ustedes.

Con respecto a la propuesta en sí, creo que es fundamental asumir que estamos ante situaciones nuevas, complejas y variadas, que se resisten a soluciones generalizadas simples, ni fáciles, por lo tanto todo intento de solución que emerja de la particularidad y peculiaridad de cada país es digna de ser tomada en cuenta.

\section{PLANTEAMIENTO DEL PROBLEMA}

Analizar el mundo actual es una tarea difícil por el desconocimiento frente a los grandes cambios producidos en el escenario nacional y mundial que hacen necesario escudriñar la realidad para reconocer el presente y pensar mejor el futuro al que nos acercamos a paso rápido.

La crisis económica global actual, que empezó aquel jueves que las economías del mundo capitalista se hundieron en la recesión y cuyas consecuencias se vienen manifestando a través de la caída del consumo, despidos laborales masivos, derrumbe de los precios del petróleo y de las materias primas, devaluación de las monedas y revaluación imparable del dólar, colapso financiero con quiebra de bancos, crisis crediticia, incremento de precios de los alimentos y la energía; se contagió rápidamente al resto de la economía mundial.

Este tsunami recesivo proyecta la agudización del proceso con baja de consumo como efecto del derrumbe globalizado; situación que nos plantea grandes retos como país, ya que la solución a esta crisis económica mundial sólo puede pensarse de forma individual, teniendo en cuenta las peculiaridades y particularidades de cada país.

Ante este escenario hostil de la economía mundial, el punto es ¿cuál sería nuestro salvavidas?, ¿cuál es el rol que debe asumir el Estado?, ¿cuál es el papel de las MYPE en 
medio de tan desfavorable contexto?, ¿qué es lo que debemos hacer como sociedad civil? La respuesta a ello: trabajo en equipo. Asociatividad.

Si bien es cierto no existen recetas mágicas, ni manuales a seguir, nuestro planteamiento pretende ser un pequeño aporte a esta situación.

Bien, un primer paso lo puede dar el Estado al tomar medidas de austeridad, gastando en lo que realmente nos ayude a sobrevivir a esta etapa y que a su vez garantice la estabilidad social. El hecho de que el Estado tenga un norte ante esta crisis ayudará a generar un clima de tranquilidad en la población. Esto puede significar que el Estado sólo cubra las necesidades básicas salud, educación y vivienda, $y$ reoriente sus esfuerzos en financiar los sectores productivos y las MYPE, tomando medidas concretas para salvarlos de esta crisis y que como efecto rebote estos nos terminen salvando a nosotros.

En cuanto a los sectores productivos y las MYPE, tendrán como principio promover la asociatividad y competitividad para lograr su inserción en el actual mercado global, con todas las implicancias que ello signifique.

Es bien sabido que en nuestro país, las MYPE generan aproximadamente el $80 \%$ del empleo, generan el $40 \%$ del producto bruto interno y aportan el $45 \%$ de la riqueza generada, entre otros. En pocas palabras, las MYPE en lo referente a temas como la generación de empleo, mejora de la competitividad, inclusión de la cooperación y asociatividad, promoción de las exportaciones y sobre todo el crecimiento de nuestro país, son un elemento clave para enfrentar el actual contexto recesivo.

Entonces, las micro y pequeñas empresas son importantes para lograr un tipo de desarrollo con inclusión social. La necesidad de reorientar esfuerzos para protegerla y po- tenciarla se basa, pues, en esta capacidad que tienen de democratizar oportunidades.

Si las MYPE ingresan al mercado para generar empleo y oportunidades a los pobladores del país, debemos entonces velar por su efectiva inserción en el mercado bajo el contexto actual sabido.

Ahora bien, como sociedad civil nos correspondería entonces, organizarnos en diferentes tipos de asociaciones - al estilo argentino - es decir, unirnos para tener mayor fuerza y poder superar la coyuntura que vivimos.

Para enfrentar adecuadamente el actual mercado exterior es necesario que las MYPES, el Estado, la sociedad civil y el sector académico concierten y producto de ello realicen un gran Proyecto Nacional de Desarrollo, para poder reencauzar nuestra economía. De esta manera, más que un reto, el panorama actual se vislumbra como una oportunidad para lograr un desarrollo autónomo y estructurar de una vez por todas ese Proyecto Nacional de Desarrollo que involucre a todos los sectores y que sea producto de las condiciones reales que tenemos como país.

Debo resaltar que el desarrollo de esta propuesta debe tener como base al capital social, es decir al capital humano.

En tal sentido, se hace necesario que este capital humano sea capaz de construir redes internas y externas que sean proactivas, flexibles y prestas a alinearse con los requerimientos de la nueva economía mundial. Por lo tanto, lo pertinente sería convertir a las micro y pequeñas empresas, no sólo en consorcios para cubrir alguna eventual demanda de producción a gran escala, sino convertirlas en redes de apoyo y solidaridad que interactúen permanentemente en beneficio de nuestra economía.

Esta propuesta se sustenta en el hecho de que las micro y pequeñas empresas han 
sido las más golpeadas por la crisis, y ello se debe a sus limitaciones - tanto internas como externas - precisamente derivadas de su limitada escala y sus débiles relaciones de articulación y colaboración, que les impiden explotar su verdadero potencial.

Dentro de este planteamiento, la expresión "redes sociales" es sinónimo de herramienta para la mejora mediante el uso de activos sociales preexistentes.

Desde tiempos remotos, en nuestro país existió una forma de solidaridad y apoyo mutuo, basada en un conjunto de lazos diádicos, todos del mismo tipo entre una serie de actores que pueden ser personas $u$ organizaciones. Pero, para entender mejor este planteamiento analicemos más de cerca lo que son las redes sociales.

\section{EL ANÁLISIS DE REDES SOCIALES}

El análisis de redes sociales ha prosperado de forma extraordinaria en el mundo durante los últimos años, su empleo ha abarcado varios campos de la investigación y para muchos investigadores es el método que hacía falta para estudiar determinados fenómenos de los que no se podía dar cuenta con los métodos ya existentes; ¿pero en qué consiste tan, relativamente, novedoso método?

Pues bien, el análisis de redes sociales consiste en el estudio sistemático de las estructuras sociales. Panfichi afirma que “... el análisis de redes se basa en los vínculos que los individuos establecen en su vida cotidiana buscando satisfacer una amplia gama de necesidades como bienes materiales, empleo, información crucial para la sobrevivencia, apoyo emocional, identidad, cohesión política, o mecanismos informales de control social.

Existen múltiples tipos de vínculos, los cuales se establecen en base a un mutuo reconocimiento de un conjunto de obligaciones y derechos entre las personas que forman parte de una red. Los derechos y obligaciones pueden estar organizados a través de una matriz de intercambios horizontales o verticales...."

La dirección de estos intercambios nos ayuda a entender la naturaleza de una red particular, así como las relaciones entre sus miembros; la naturaleza de estas redes es lo que determina el éxito o fracaso de ciertos mecanismos de sobrevivencia, formas de organización social y económica, y prácticas políticas de los pobres, por ejemplo.

Los recursos materiales, valores y la información distribuida a través de las redes sociales, constituyen factores centrales en el desarrollo de solidaridades sociales, económicas y políticas entre sus miembros. Estas solidaridades son la base de acciones sociales y políticas, las cuales pueden tomar una gran variedad de formas.

Muchos sociólogos señalan que ciertas redes sociales, en especial aquellas organizadas para la sobrevivencia, pueden ser el "eslabón perdido" capaz de conectar los niveles macro y micro de análisis; y la experiencia individual con la acción colectiva.

En cuanto a las técnicas, es de notar que en las últimas décadas se ha producido un formidable desarrollo de las técnicas de análisis estadístico de datos relacionales, paralelo al diseño de programas informáticos para el estudio y representación de redes.

Pero, el análisis de redes sociales no se limita a un conjunto de indicadores de cen-

1 Panfichi, Aldo: “Los pobres de las ciudades latinoamericanas: balance y perspectivas teóricas”. En: Revista de Sociología No 9. Fac. CC.SS. UNMSM. Perú. 1994. 
tralidad y agrupación, sino que es un instrumento para la investigación de sistemas sociales, especialmente apropiado para poner en relación los niveles micro y macro-social.

Hoy en día, el concepto de redes sociales abarca una amplia gama de disciplinas científicas, se utiliza por ejemplo para explicar temas tan diversos como el acceso de los individuos al mercado de trabajo, la distribución del poder, el proceso de contagio de enfermedades, las relaciones entre organizaciones productivas, la constitución de comunidades virtuales, las redes transversales frente a redes cohesivas como base del capital social, las redes personales de apoyo social y autoayuda, la estructura del poder político, las conexiones inter-organizacionales, el software de análisis estadístico y representación gráfica, etc.

Los agentes susceptibles de ser estudiados bajo este enfoque son muy diversos: individuos, empresas, instituciones, regiones, organizaciones, etc., pudiendo centrarse el estudio en el análisis de las relaciones mantenidas por uno o varios agentes, o en las estructuras relacionales que definen determinados grupos o colectivos.

Para esta nueva percepción de análisis, las relaciones sociales no están necesariamente delimitadas por la dimensión espacial o territorial, ya que los individuos pueden construir sus propias redes de vínculos sociales, políticos o económicos, sin necesidad de socializar con sus vecinos.

Así también, como lo afirma Enrique Vázquez $^{2}$, por ejemplo, para los pobres, las redes sociales posibilitan el acceso al mercado formaly les ayudan a repotenciar sus capacidades económicas; la red social es un mecanismo informal donde la confianza y la integración social son elementos fundamentales.
Como vemos el concepto de red social resulta decisivo y es en ese sentido que puede considerarse como parte del capital social.

El análisis de redes, al partir de un reposicionamiento de las técnicas de análisis estructural, es sin duda alguna, una alternativa metodológica que permite una mejor interlocución entre las ciencias económicas y las ciencias sociales, fundamental para la comprensión de un mundo industrial y del trabajo cuyos principales paradigmas están en plena reformulación, - debido al desenlace que ha tenido - así como para orientar con mayor claridad las políticas públicas de alcance nacional y local relativas al contexto de crisis actual.

La perspectiva de redes implica en este campo, la aceptación de un enfoque metodológico basado en el estudio de sistemas socioeconómicos, formadas por las relaciones mantenidas entre los actores u organizaciones que los componen.

La elusión de las relaciones sociales supone ignorar el carácter social de los agentes económicos, es decir, del comportamiento humano, que constituye la base de los sistemas económicos al nivel más micro, que es el que nos interesa en este caso.

Sin embargo, la realidad nos enseña que todos los agentes económicos son actores sociales que adoptan sus decisiones, inmersos en una red de relaciones sociales que proporciona oportunidades, pero también restricciones, dependiendo de cuál sea la posición que ocupen en ella.

Aplicar el análisis de redes sociales enriquece toda investigación y programas de actividades que tengan en el desarrollo de las relaciones y lazos sociales su foco de atención.

2 Vásquez H., Enrique: Introducción. En: ¿Cómo reducir la pobreza y la inequidad en América Latina? Centro de Investigación de la Universidad del Pacífico. Programa Latinoamericano de Políticas Sociales. Perú. 2000. 
Uno de los principales aportes de la teoría de redes es el señalado por Enmanuelle Barozet ${ }^{3}$ : “... La teoría de redes ha ayudado a los actores civiles a tomar conciencia de la importancia que representa el 'capital social' para conseguir metas diversas..."4.

Finalmente, coincidimos con Castells (1999), en que la nueva economía globaliza$\mathrm{da}$, con su desarrollo industrial heterogéneo que comienza a volver los ojos al humilde terreno de lo local, es esencialmente una economía de redes, entonces podríamos decir que el análisis de redes sociales es la herramienta adecuada para comprender el mundo actual en que vivimos, y el método apropiado para insertarnos en la nueva economía con éxito.

Se entiende así a las redes sociales como un modo de impulsar el fortalecimiento de la trama vital y de la sociedad; y con ello se plantea la posibilidad de abordar desde este pensamiento tanto las redes personales como organizacionales.

Pero en general, se ha logrado demostrar que es posible ganar en conocimiento, compartiendo información y trabajando de forma cooperativa.

Investigadores provenientes de diversos campos disciplinares, desde su propia perspectivas incorporan el análisis de redes sociales para visualizar los vínculos; y construir y contrastar hipótesis acerca de los patrones emergentes de la interacción a distintos niveles de organización. Todos compartimos el interés por aceptar el desafío de obtener nuevas explicaciones de los fenómenos en estudio, que articulen las preguntas que surgen del análisis de los datos empíricos con la construcción de explicaciones más generales.
Estos trabajos combinan la aplicación de algunos conceptos de la teoría de redes: redes totales, capital social, patrones relacionales emergentes, análisis estructural; con el manejo de instrumentos formales y computarizados: delimitación de variables, cálculos de frecuencias, correlaciones estadística, estimaciones de medidas de centralidad y graficación de redes.

Vinculado al análisis de redes sociales, siempre encontraremos el concepto de capital social, que resulta trascendente en la comprensión de esta temática.

El capital social es un término que hace referencia a las normas, instituciones y organizaciones que promueven confianza, ayuda recíproca y cooperación; y con ello, se atribuye que puede reducir costos de transacción, producir bienes públicos y facilitar la constitución de organizaciones favorables para el desarrollo.

El capital social se concentra en los beneficios que otorga la disponibilidad de una red de relaciones sociales durables y de los recursos asociados a ellas resultando de interés las instancias intra-organizacionales e inter-organizacionales con estructuras de interacción particulares.

En este sentido, se ha propuesto una visión integrada entre el enfoque del capital social y el de redes.

Por ello, intento entender las dinámicas que ligan a los individuos unos con otros, a las organizaciones unas con otras y cómo estas relaciones establecen roles y mantienen o cambian las estructuras de la sociedad, y específicamente cuál puede ser su aporte en el actual contexto económico mundial y de nuestro país.

3 Doctora en Sociología de la Escuela en Altos Estudios en Ciencias Sociales de París. Profesora Instructora en el Instituto de Ciencias Políticas.

4 Barozet, Enmanuelle: "De la elaboración teórica a la aplicación de casos: las redes y su uso social". Ponencia. En: Proyecto de la Fundación Nacional de Superación de la Pobreza Desde la Experiencia. 2005. 


\section{Redes Sociales y MYPE}

Teniendo en cuenta la coyuntura mundial, aceptémoslo, la micro y la pequeña empresa difícilmente sobrevivirán, ni mucho menos lograrán un alcance global actuando individualmente. Vemos así que las micro y pequeña empresas son las que más sufren los efectos de la crisis global.

En un mercado cada día más competitivo, la asociación y coordinación entre las micro y pequeñas empresas es una necesidad vital y además urgente. $Y$ es en este punto donde recordamos que cada problema contiene su propia solución; frente a la aguda crisis de la economía mundial, que se ha extendido a nuestro país, que pone en riesgo factores hasta de gobernabilidad y que ha golpeado a la micro y pequeña empresa, aún no con todo su poder; la solución surge de la fuerza creativa y del ingenio - tantas veces resaltado- de nuestra propia gente, que desde tiempos pasados se asoció para realizar labores comunes.

Emerge entonces, la fuerza de la asociatividad como ese salvavidas que esperábamos, como una respuesta renovada de aprovechar el capital humano y los recursos endógenos como el eje que nos permita establecer un nuevo estilo de desarrollo autónomo basado en las potencialidades de las economías locales y bajo un enfoque de redes sociales.

Se trata, pues, de concentraciones de pequeñas empresas que se organizan en función de una misma actividad y forman una primera red que es parte de otra mayor y que tiene como meta la exportación de nuestros productos al mundo.

Si bien es cierto, esta idea del emprendimiento colectivo no es novedosa, pienso que bajo un rostro humano este enfoque de redes puede resultar beneficioso, porque la coyuntura económica actual simboliza un reto para la gestión de las micro y pequeñas empresas, $y$ a su vez las induce a asumir retos, para tener más posibilidades de vencer y elevarse a las características nefastas del actual mercado mundial. Por ello, con este modelo se pretende revalorizar lo propio, lo local, lo interno, como fuente de desarrollo y de crecimiento, $y$ en este contexto, las MYPES tendrán un papel fundamental en el desarrollo nacional, regional y sobre todo local, promoviendo su competitividad, productividad y asociatividad, en la perspectiva de mejorar su participación en la nueva economía del mercado global.

\section{El enfoque de redes y la crisis recesiva mundial}

Ahora bien, es interesante observar cómo toda situación está atravesada por este enfoque de redes, inclusive la globalización —red mundial globalizada- y la crisis mundial en la que ha degenerado - crisis recesiva global- no escapan de ella, ya ilustran cómo esta crisis afecta todo el tejido de la red económico-financiera global, hasta llegar a nosotros.

Entonces, la globalización de la economía mundial significa que nuestros bancos, corporaciones y todo tipo de organización económico-financiera que eran parte de la economía global, de la red financiera global, caen ante la crisis de ésta, porque no son otra cosa más que "sucursales" de las economías de Estados Unidos y de Europa, y lo que sufren es un efecto espejo de los grandes mercados globales de dinero, controlados por el "gran capital financiero" concentrado en estos países que han planetarizado la crisis, $\mathrm{y}$ todo porque nuestro sistema financiero $\mathrm{y}$ económico-productivo está regido por las mismas reglas de juego que tienen los bancos y corporaciones industriales que ahora están en crisis. 
Estos patrones del capitalismo financiero internacional han fracasado y está comprobado que son incapaces de ofrecer una solución a tan grave crisis, pues a este sistema sólo le interesa su propia salvación, y no la de los países con sus sectores productivos y sociales que sufren sus efectos.

Lo que aquí se ha planteado es una perspectiva de solución que parte de nuestra propia realidad y que puede o no ser correcta, pero es un intento endógeno de solución frente a esta crisis y no una copia de algún "plan anticrisis de rescate" fracasado ya en su propia tierra.

En esta tarea queda mucho por investigar, y como casi en todo este enfoque de redes debe tener limitaciones, pero su aplicación al tema de la micro y pequeña empresa resulta provocativo.

\section{CONCLUSIONES}

La principal limitación que enfrentan las micro y pequeñas empresas en el actual contexto recesivo mundial, se deriva de su limitada escala y de sus débiles relaciones de articulación y asociatividad.

Estas limitaciones pueden ser suplidas por la creación de redes sociales que tengan como base el capital humano que, a su vez, se sustentaría en vínculos de asociación, colaboración, reciprocidad, etc., para desarrollar un determinado trabajo.

Este capital humano debe ser capaz de construir redes internas y externas que sean proactivas, flexibles y prestas a alinearse con los requerimientos de la nueva economía global.

El análisis de redes se basa en los vínculos que los individuos establecen en su vida cotidiana buscando satisfacer una amplia gama de necesidades como bienes materiales, empleo, información crucial para la sobreviven- cia, apoyo emocional, identidad, cohesión. Los recursos materiales, valores y la información distribuida a través de las redes sociales constituyen factores centrales en el desarrollo de solidaridades sociales, económicas y políticas entre sus miembros.

La solución a esta crisis económica mundial sólo puede pensarse de forma individual, teniendo en cuenta las peculiaridades y particularidades de cada país.

En tal sentido, la nueva economía ha vuelto los ojos al terreno de lo local, y en él, la economía de redes es la clave.

Dentro de la actual coyuntura mundial, la asociatividad y la coordinación entre las micro y pequeñas empresas es una necesidad vital para su sobrevivencia y el de nuestra economía.

El Estado debe reorientar sus esfuerzos en financiar los sectores productivos y las MYPE, tomando medidas concretas para salvarlos de esta crisis y que como efecto rebote éstos nos terminen salvando a nosotros.

En este contexto, las MYPE tendrán como principio promover la asociatividad y competitividad de las mismas para lograr su inserción en el actual mercado global, con todas las implicancias que ello signifique.

Las MYPE son importantes porque logran un tipo de desarrollo con inclusión social, democratizando oportunidades.

Más que un reto, la coyuntura actual se presenta como una oportunidad de estructurar un Proyecto Nacional de Desarrollo, que reencauce nuestra economía y logre un desarrollo autónomo producto de las condiciones reales que tenemos como país.

\section{REFERENCIAS BIBLIOGRÁFICAS}

1. Aliaga, L. (2002). "Racionalidad económica y redes sociales, las paraditas y su 
mercado". Revista Investigaciones Sociales. Perú: Instituto de Investigaciones Histórico Sociales. No 9.

2. Barozet, E. (2005). "De la elaboración teórica a la aplicación de casos: las redes y su uso social”. En Proyecto de la Fundación Nacional de Superación de la Pobreza Desde la Experiencia.

3. Borgatti, S. \& Collage, B. (2003). Conceptos Básicos de Redes Sociales.

4. Molina, J. (2001). El análisis de redes sociales. Una introducción. España: Ediciones Bellaterra.

5. Panfichi, A. (1994). "Los pobres de las ciudades latinoamericanas: balance $\mathrm{y}$ perspectivas teóricas”. Revista de Sociología. Vol. 8, No 9. Perú: Fac. CC.SS, UNMSM.

6. Vásquez, E. (2000). ¿Cómo reducir la pobreza y la inequidad en América Latina? Perú: Centro de Investigación de la Universidad del Pacífico.

7. Villegas, C. (2004). Emprendimientos colectivos: Las MYPES y las innovaciones para su inserción en la nueva economía. www.gestiopolis.com/recursos2/documentos/fulldocs/emp/empcolec.htm. (visitado el 12-04-09)

8. http://www.analytictech.com/networks/introduccion2.pdf (visitado el 3103-09). 